\title{
Different expression patterns of duplicated PHANTASTICA-like genes in Lotus japonicus suggest their divergent functions during compound leaf de- velopment
}

\author{
Jiang Hong LUO ${ }^{1}$, Jun YAN ${ }^{1}$, Lin WENG ${ }^{1}$, Jun YANG ${ }^{1}$, Zhong ZHAO ${ }^{3}$, Jiang Hua CHEN ${ }^{1}$, Xiao He HU ${ }^{1}$, Da \\ $\mathrm{LUO}^{1,2, *}$
}

\begin{abstract}
${ }^{1}$ National Laboratory of Plant Molecular Genetics, Institute of Plant Physiology and Ecology, Shanghai Institutes for Biological Sciences, and Graduate School of the Chinese Academy of Sciences, Chinese Academy of Sciences, 300 Fenglin Road, Shanghai 200032, China

${ }^{2}$ School of Life Science and Biotechnology, Shanghai Jiao Tong University, 1954 Huashan Road, Shanghai 200030, China ${ }^{3}$ Department of Biochemistry, School of Life Sciences, Fudan University, Shanghai 200433, China
\end{abstract}

\begin{abstract}
Recent studies on leaf development demonstrate that the mechanism on the adaxial-abaxial polarity pattern formation could be well conserved among the far-related species, in which PHANTASTICA (PAHN)-like genes play important roles. In this study, we explored the conservation and diversity on functions of $P H A N$-like genes during the compound leaf development in Lotus japonicus, a papilionoid legume. Two PHAN-like genes in L. japonicus, LjPHANa and LjPHANb, were found to originate from a gene duplication event and displayed different expression patterns during compound leaf development. Two mutants, reduced leaflets1 (rell) and reduced leaflets3 (rel3), which exhibited decreased adaxial identity of leaflets and reduced leaflet initiation, were identified and investigated. The expression patterns of both $L j P H A N \mathrm{~s}$ in rel mutants were altered and correlated with abnormalities of compound leaves. Our data suggest that $L j P H A N a$ and LjPHANb play important but divergent roles in regulating adaxial-abaxial polarity of compound leaves in L. japonicus.
\end{abstract}

Keywords: adaxial identity, LjPHANa, LjPHANb, gene duplication, rel mutants, Lotus japonicus.

\section{INTRODUCTION}

The leaf organs of higher plants can be classified as simple or compound leaves. Compound leaves are found in distantly related groups, and differ from simple leaves in that each petiole bears multiple leaflets lacking auxiliary buds $[1,2]$. Most leaves possess three-dimensional polarities that can be dissected into the adaxial-abaxial, proximodistal and mediolateral polarities, among which the adaxial-abaxial polarity is established early in leaf primordia and determines the leaf expansion [3]. Many regulators are involved in adaxial-abaxial patterning of Arabidopsis leaves, including the adaxial identity genes $P H A N$ TASTICA, PINHEAD, ARGONAUTE 1, PHABULOSA and PHAVOLUTA [4-7]; the abaxial identity genes YABBYS

\footnotetext{
*Correspondence: Da LUO

Fax: +86-21-5492-4106;

E-mail: dluo@sibs.ac.cn
}

and KANADIs $[8,9]$.

PHANTASTICA was the first gene found to be required for establishment of adaxial-abaxial polarity; when PHAN was mutated in Antirrhinum, the adaxial identity of leaves was lost and needle-like leaves were formed $[5,10]$. Orthologs of PHAN have been reported in many species, such as ROUGH SHEATH2 (RS2) in maize $[11,12]$ and Asymmetrical Leaves 1 (AS1) in Arabidopsis [13, 14]. PHAN genes are also showed to be involved in proximodistal elaboration [15] and palisade development [16]. Another important function of $P H A N$ genes is responsible for suppressing expression of class 1 KNOTTED1-like homeobox I $(K N O X I)$ genes in leaf primordia, which are often expressed in meristems. Loss of functional PHAN genes causes ectopic expression of KNOXI genes and ectopic meristematic activity in the leaf primordia, which result in lobed leaves or ectopic growth on leaf $[11-13,16,17]$. The complementary expression patterns between PHAN and KNOXI were also found in leaves of Selaginella kraussinna, 
a lycophyte with mcrophyllous leaves [18], which suggested that PHAN-KNOXI interaction is a common mechanism in determining leaf development.

In compound leaf development, $P H A N$ genes were also found to reserve the function in building the adaxial domain in both compound leaf primordia and leaflet primordia; proper expression of those genes is required for correct patterning of leaflet arrangement that is used for proper differentiation between pinnate and palmate compound leaves in eudicots [19]. On the other hand, over-expression of KNOXI genes caused lobed leaf phenotype in Arabidopsis as well as enhanced complexity of compound leaves in tomato, suggesting that $K N O X I$ genes might play an important role in forming a compound leaf [20-23]. Further studies revealed that re-establishment of KNOXI gene expression in the compound leaf primordia was responsible for the maintenance of meristematic activity and the initiation of leaflets, which was conserved in the compound leaf development of most vascular plants, with the exception of legumes [24, 25]. In tomato, the expression domain of the PHAN genes overlaps with that of the $K N O X I$ genes, and their proper expression balance appears to be required for accurate regulation of compound leaf development [26, 27].

In legumes, most studies on compound leaf development have been carried out in pea. It has been shown that UNIFOLIATA (UNI), an ortholog of LEAFY, rather than $K N O X I$ genes play an important role in maintaining the meristematic activity in compound leaf primordia $[24,28]$. $S T P$, an ortholog of UNUSUAL FLORAL ORGANS involved in floral development, also regulates the complexity of compound leaves in pea [29]. CRISPA (CRI), the pea ortholog of $P H A N$, is believed to be required for adaxialabaxial polarity of leaflets and suppress expression of $K N O X I$ genes in compound leaf primordia in pea [30]. The relation between $C R I$ and $K N O X I$ is similar to that in Arabidopsis, indicating that mechanism controlling pea compound leaves is different from that in tomato. However, compound leaves in pea are highly specialized because their lateral organs show distinct identities: stipules, leaflets and tendrils [30], and this raises the question about the function of $P H A N$-like genes and their interaction with $K N O X I$ genes in other papilionoid legumes with relatively simple form of compound leaf.

In this study, we choose L. japonicus as the experiment system, whose compound leaf typically consists of five visible leaflets. We herein isolated two $P H A N$-like genes, $L j P H A N a$ and $L j P H A N b$, and three KNOXI genes, LjKN1, LjKN2 and LjKN3, in L. japonicus. Two mutants with abnormal compound leaves, reduced leaflets 1 (rell) and reduced leaflets 3 (rel3), were identified, which exhibited decreased adaxial identity of leaflets and reduced leaf- let initiation. The expression patterns of $\angle j P H A N \mathrm{~s}$ and $L j K N \mathrm{~s}$ were examined in wild type as well as in rel mutants. Our results suggest that duplicated $P H A N$-like genes have divergent functions in regulating compound leaf development and possibly act together to repress the KNOXI gene expression in L. japonicus compound leaves.

\section{MATERIALS AND METHODS \\ Plant material and growth conditions}

The rell and rel3 mutants used for the analysis were M4 segregated offspring with mutant phenotypes. The wild-type L. japonicus (Gifu-B129 ecotype), rell and rel3 mutants were grown in a growth chamber at $22^{\circ} \mathrm{C}$ on a $16 \mathrm{~h}: 8 \mathrm{~h}$ light:dark cycle.

\section{Isolation of homologs and sequence analysis}

RNA was prepared from vegetative shoots of L. japonicus (GifuB129 ecotype) and soybean (Jilin21 variety) using the RNeasy Plant Mini Kit (Qiagen). cDNA was synthesized from total RNA using the SL0066 primer (5'gac tcg agt cga cat cgt $\mathrm{t}_{16} 3^{\prime}$ ) and AMV retrotranscriptase enzyme (Promega). LjPHANa and $L j P H A N b$ were amplified by 3'-RACE using degenerate primers SL0445 [5' cac gg(a/ $\mathrm{g} / \mathrm{c})$ aac aa $(\mathrm{a} / \mathrm{g}) \operatorname{tgg} \mathrm{a}(\mathrm{a} / \mathrm{g}) \mathrm{aa}(\mathrm{a} / \mathrm{g})$ at $(\mathrm{t} / \mathrm{c})$ gc3'] and SL0446 [5'aag tgg tgg $\left.\mathrm{ga}(\mathrm{a} / \mathrm{g}) \mathrm{gt}(\mathrm{a} / \mathrm{t} / \mathrm{g}) \mathrm{tt}(\mathrm{c} / \mathrm{t}) \mathrm{aa}(\mathrm{a} / \mathrm{g}) \operatorname{gag} 3^{\prime}\right]$, respectively, as the $5^{\prime}$ primer and SL0065 (5'gac tcg agt cga cat cg3') as the 3' primer. The full length ORF of $L j P H A N a$ was amplified from cDNA with specific primers SL0711 (5'cta gag aat ggc atc tcg tgt c3') and SL0712 (5'tgt aaa act gga ggt tag gca c3'), and the full length ORF of $L j P H A N b$ was amplified with SL0713 (5'cac aga aat atg att agt tca aga t3') and SL0519 (5'gaa tag ata gga aat ttg cac aa3'). GmPHANa was amplified from cDNA with specific primers SL0913 (5'ctt gga aat gaa aga tag gca a3') and SL0914 (5'taa ttt aac tga cct gtg tga gct a3'), and GmPHANb was amplified with SL0915 (5'ctt gga aat gaa aga gag gca3') and SL0916 (5'tag tca gaa gag cac caa tat cca t 3'). LjKN1 was amplified from cDNA using SL0161 (5'atc acc gtc tct tgg cag c $3^{\prime}$ ) as the 5' primer, and SL0065 and SL0162 (5'tgg-gta-agg-cca-ttt-gta-atg 3') as the 3' primers. LjKN2 was amplified by SL0512 [5'gc(t/c)-tac-atg-ga(c/t)-tg(c/t)-caaaag3'] as the 5' primer, and SL0065 and SL0513 [5'gaa cca (ga)tt (g/a) tt tat $\left.\operatorname{ttg}(\mathrm{c} / \mathrm{t}) \mathrm{tt}(\mathrm{t} / \mathrm{c}) \operatorname{tg} 3^{\prime}\right]$ as the 3' primers. LjKN3 was amplified by SL0514 [5'atc aa(a/g) gc(c/t) aaa atc $g(\mathrm{c} / \mathrm{t})(\mathrm{c} / \mathrm{t})$ tci ca(c/t) cct3'] as the 5 ' primer, and SL0065 and SL0515 [5'gca t $(\mathrm{g} / \mathrm{a}) \mathrm{t} \mathrm{t}(\mathrm{c} / \mathrm{t}) \mathrm{t}$ cig aig $\mathrm{g}(\mathrm{c} / \mathrm{t}) \mathrm{t}$ tcc aat $\left.g 3^{\prime}\right]$ as the 3 ' primers. The PCR products were cloned into the pGEM-T easy vector (Promega) and sequenced. Sequence analyses were carried out, using the GenBank database along with the Bioedit 6.0 [31], ClustalX 1.83 [32], and TreeView 1.6.6 [33] software packages.

\section{Genomic southern blotting}

Genomic DNAs of L. japonicus (Gifu-B129 ecotype), soybean (Jilin21 variety), Medicago trucatula (Jemalong ecotype) and pea (Hortense Poir. variety)were digested with HindIII, EcoRI, EcoRV, and double digested with combination of any two enzymes mentioned as above, or with BamHI. The digested DNA fragments were transferred onto Hybond $\mathrm{N}+$ nylon membranes by alkaline transfer method. The probe was labelled by random primer labelling (Promega) and GmPHANa cDNA was used as template. The membranes were hybridized at $55^{\circ} \mathrm{C}$ and washed at $55^{\circ} \mathrm{C}$ with $0.1 \times \mathrm{SSC}$ and $0.1 \%$ SDS after $1 \times \mathrm{SSC}$ and $0.1 \% \mathrm{SDS}$, and then exposed to phosphor image plate or X-film. 


\section{Construction of transgenic Arabidopsis}

$L j P H A N a(1.1 \mathrm{~Kb})$ and $L j P H A N b(1.2 \mathrm{~Kb})$ cDNA fragments were inserted into the pMON530 binary T-DNA vector downstream of the $35 S$ promoter (generously gifted by the Lab of Dr. Hai HUANG). Plasmids $35 S:: L j P H A N a$ or $35 S:: \angle j P H A N b$ were introduced into Arabidopsis plants (Landsberg erecta) by Agrobacterium-mediated transformation. Sixteen $35 S: \because L j P H A N a$ transgenic lines and ten $35 S::$ $\angle j P H A N b$ transgenic lines were obtained after $K m^{\mathrm{r}}$ selection on MS medium; they were transferred to soil and grown in a growth chamber at $22^{\circ} \mathrm{C}$ on a $16 \mathrm{~h}: 8 \mathrm{~h}$ light:dark cycle. Transgenic plants were verified by PCR with specific primers SL0711 and SL0712 for LjPAHNa and SL0713 and SL0519 for LjPAHNb.

\section{Scanning electron microscopy and histology}

The samples used for SEM were treated as previously described [34] and examined at an acceleration voltage of $15 \mathrm{kV}$ on Hitachi S2460. The tissues for histology were fixed and sectioned as described previously [35]. Sections of leaflet blades were stained by Toluidine blue.

\section{In situ hybridization}

Material for in situ hybridization was fixed, sectioned, and hybridized to digoxigenin-labeled RNA probes as described previously [36]. Templates for the $L j P H A N a$ - and $L j P H A N b$-specific probes were obtained from amplification with specific primers [for $L j P H A N a$, SL516 (5'tcg cgg aga agc tag tga aa3') and SL0517 (5'atc cca atg gaa atg gaa cat a3'); for $L j P H A N b$, SL518 (5'taa gga gaa gca gca gag aga3') and SL0519 (5'gaa tag ata gga aat ttg cac aa3')]. The partial cDNAs of $L j K N 1$ and $L j K N 3$ were used directly as templates.

\section{RESULTS}

Isolation of $\boldsymbol{P H A N}$-like genes in $\mathbf{L}$. japonicus genome $P H A N$-like genes belong to a subgroup of $M Y B$ gene family and share a conserved C-terminal domain in addition to the N-terminal MYB domain [37]. We designed degenerate primers aimed at the conserved domains and used them to amplify cDNA from the shoots of Gifu-B129. Two PHAN-like genes, $L j P H A N a$ and $L j P H A N b$, were identified in the L. japonicus genome. Comparison between the two cDNA sequences and genomic sequences of $L$. japonicus [38] revealed that $L j P H A N a$ and $L j P H A N b$ were located in a tandem cis-cluster on chromosome 3, which indicated that the two $P H A N$-like genes likely derived from gene duplication. The ORFs of $L j P H A N a$ and $L j P H A N b$ were separated by a $4 \mathrm{~kb}$ region, which did not encode another ORF and presumably contained the promoter of LjPHANb and intergenic sequences (Fig. 1A).

Analysis of the putative protein sequences showed that the N-terminal MYB domain and C-terminal domain are highly conserved in both LjPHANs (Fig. 1B). LjPHANa shared $75.8 \%$ and $70.8 \%$ identity, and $82.9 \%$ and $77.4 \%$ similarity to PsPHAN (PHAN ortholog in pea) and MtPHAN (PHAN homolog in Medicago trucatula) respectively, while LjPHANb shared 67.3\% and 66.0\% identity, and 74.2\% and $71.9 \%$ similarity to PsPHAN and MtPHAN respectively.
In contrast, LjPHANa and LjPHANb only shared $68.8 \%$ identity and $76 \%$ similarity with each other.

We next investigated whether the genomes of other legume species may contain two or more copies of the PHAN-like genes. Publicly available database searching yielded two soybean EST sequences (BF598488 and BE330204) showing high similarities with the C-termini of $\angle j P H A N a$ and $L j P H A N b$ respectively. We isolated the two $P H A N$-like genes from soybean cDNA and named them GmPHANa and GmPHANb. The results of Genomic Southern hybridization revealed that there is likely to be more than one PHAN-like gene in all four-legume species, including L. japonicus, soybean, medicago and pea (Fig. 1D). Therefore, we assume that the duplication of $P H A N$-like genes should be generally occurred in papilionoid legumes.

A phylogenic tree of PHAN-like proteins was built using the Neighbour Joining method on predicted protein sequences from the variable region between the MYB domain and the CTD domain (C-terminal conserved domain). In the resulting tree with SvARP1 (PHAN proteins from Selaginella viticulosa) as the outgroup, the legume PHANlike proteins clustered together and were divided into two large clades. The first clade included PsPHAN, MtPHAN, GmPHANa, LjPHANa and AhPHAN (PHAN-like protein from Acacia hindisii) while LjPHANb and GmPHANb belonged to the second clade. The first clade was further divided into two small sub-clades, one shared by PsPHAN, MtPHAN and GmPHANa while AhPHAN and LjPHANa rooted in another one (Fig. 1C).

Genbank Aaccession Numbers: SvARP1 (Selaginella viticulosa), AY667453; SkARP1 (Selaginella kraussiana), AY667452; RS2 (maize), AF143447; PaPHAN (Pachira aquatica), AY180135; FaPHAN (Fraxinus americana), AY180136; SaPHAN (Schefflera actinophylla), AY180133; VcPHAN (Vitex cannabifolia), AY 180132; PHAN (Antirrhinum), AJ005586; AhPHAN (Acacia hindisii), AY180134; MtPHAN (Medicago trucatula), AF308453; PsPHAN (pea), AF299140; NtPHAN (Nicotiana tabacum), AY 559043; LePHAN (tomato), AF148934; AS 1 (Arabidopsis), AF 175996; EcPHAN (Eschscholzia californica), AY228766; AfPHAN (Aquilegia formosa), AY180131; LjPHANa (L. japonicus), AY790244; LjPHANb (L. japonicus), AY790245; GmPHANa (soybean), AY790252; GmPHANb (soybean), AY790253.

\section{Expression patterns of $\boldsymbol{P H A N}$-like genes in $\boldsymbol{L}$. japonicus}

The existence of two PHAN-like genes in L. japonicus is in contrast to most other species characterized thus far, which generally contain one copy of $P H A N$-like gene [18, 19]. To explore whether the two $P H A N$-like genes have different biological functions during compound leaf development, we assessed the expression patterns of 
A

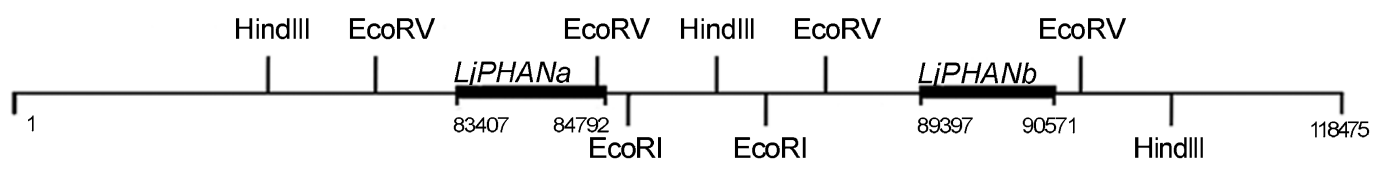

B LiT11G09(TM0020)
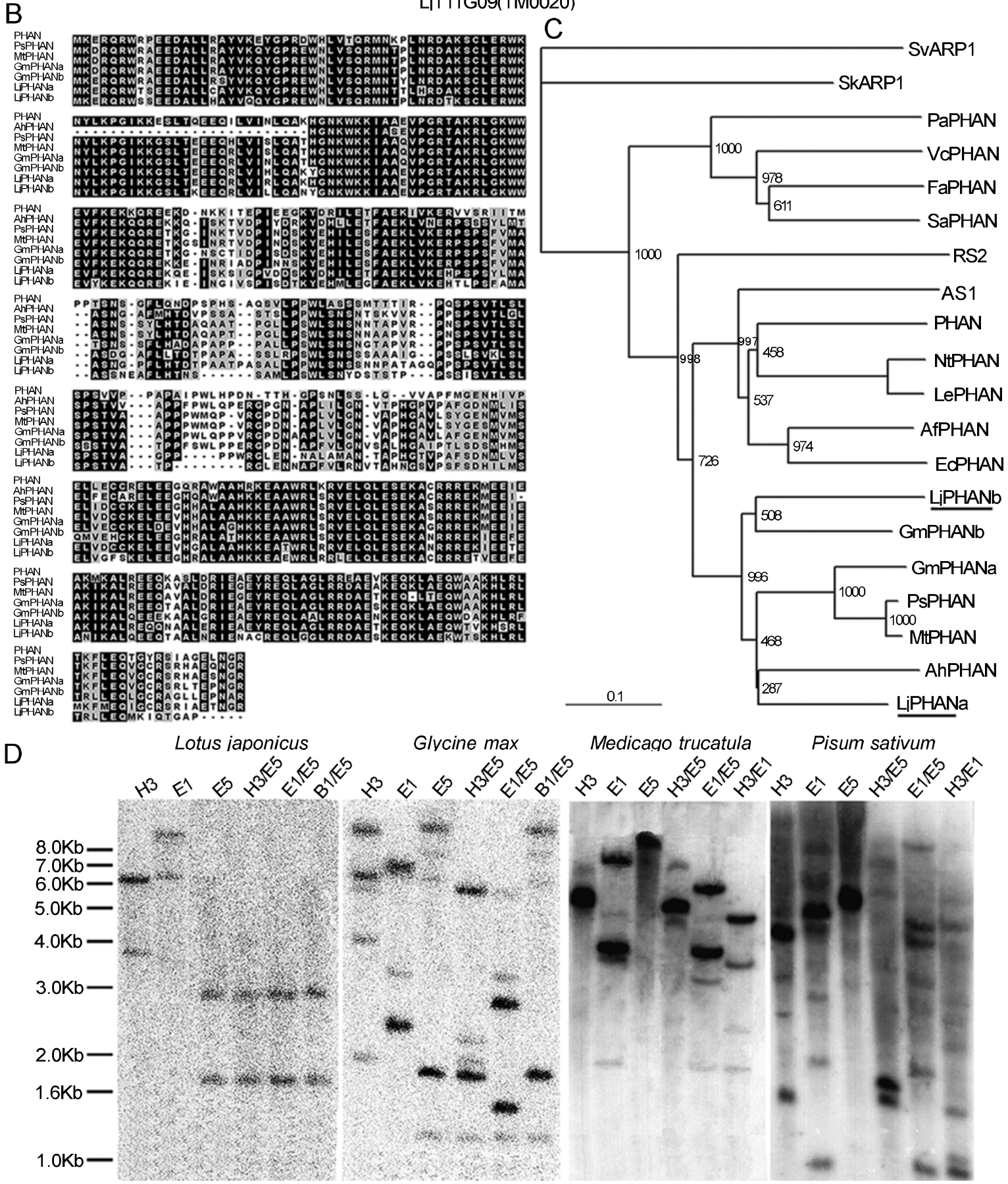

Fig. 1 Duplicated PHAN-like genes in L. japonicus. (A) Tandem arrangement of the two PHAN-like genes on LjT11G09. (B) Multiple alignment of PHAN-like proteins or fragments; residues identical in six of the proteins are shown in black. (C) A phylogenetic tree of PHAN-like proteins based on Neighbour Joining method with SvARP1 as outgroup, the analyzed sequence covering the variable region between the MYB domain and CTD domain. (D) Genomic Southern hybridization with GmPHANa cDNA as the probe. E1: EcoRI; H3: HindIII; E5: EcoRV; H3/E5: HindIII and EcoRV; E1/E5: EcoRI and EcoRV; B1/E5: BamHI and EcoRV; H3/E1: HindIII and EcoRI. 

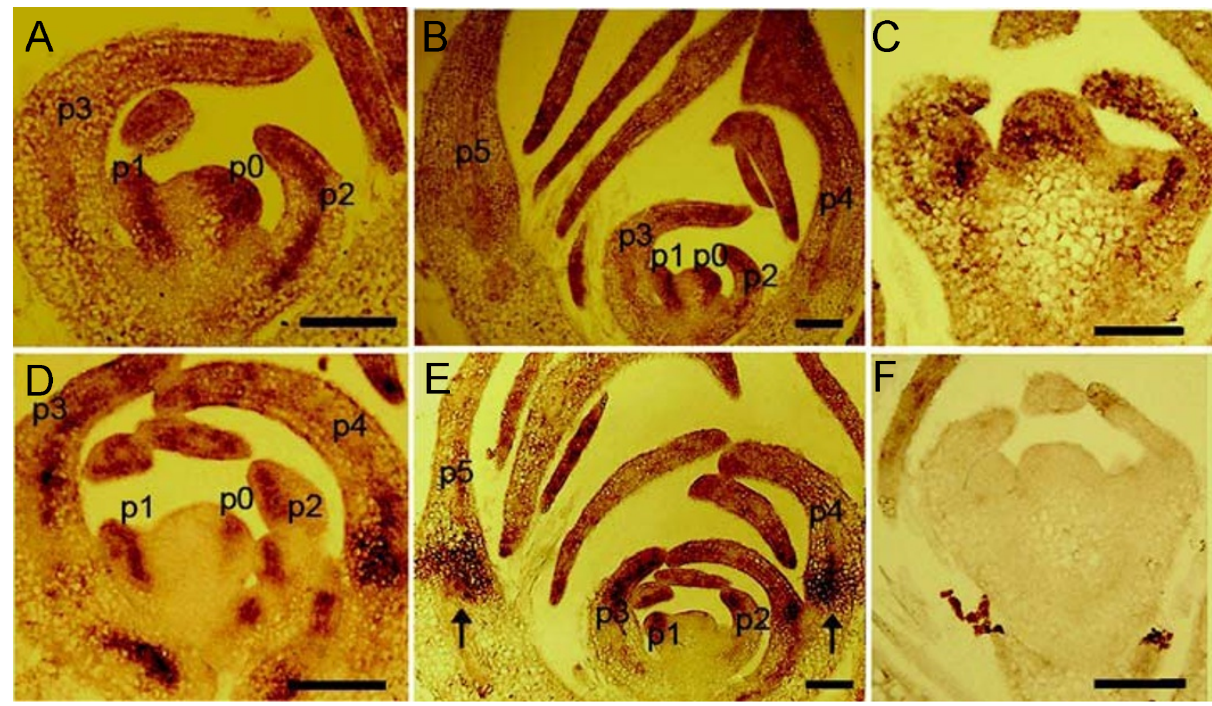

Fig. 2 Expression patterns of $L j P H A N$ genes in L. japonicus. (A-C) Expression pattern of $\angle j P H A N a$ in compound leaf primordia and leaflet primordia (A, B) and developing floral bud (C). (D-E) Expression pattern of $L j P H A N b$ in compound leaf primordia and leaflet primordia. Signal of both $L j P H A N \mathrm{~s}$ was detected in p0, and stronger expression was observed at the adaxial domains of p1 (A, D). In p4 and p5, LjPHANb was detected at the bases of leaflet primordia (as indicated by arrows in E). (F) No LjPHANb signal was detected in developing floral bud. p0-p5: compound leaf primordia at different developmental stages. Scale bar $=50 \mu \mathrm{m}$.

LjPHANa and $L j P H A N b$ by RNA in situ hybridization. $L j P H A N a$ and $L j P H A N b$ were dynamically expressed during compound leaf development in L. japonicus. The expression of both genes first appeared in the compound leaf founder cells ( $\mathrm{p} 0$ ) located in the peripheral zone of the SAM (Fig. 2A, D). Then their transcripts were detected in p1 compound leaf primordia, with stronger level in the adaxial domain (Fig. 2A, D). Therefore, LjPHANa and $L j P H A N b$ displayed similar expression patterns during the earlier phase of compound leaf development.

Later when leaflets initiated, expression pattern difference between the two $L j P H A N \mathrm{~N}$ was observed. In newly initiated leaflet primordia, $L j P H A N a$ expression was detected with stronger level at the middle region of leaflet primordia in $\mathrm{p} 2$ (Fig. 2A, B), and then dispersed in the older leaflet primordia of $\mathrm{p} 3$ and $\mathrm{p} 4$ (Fig. 2A, B). On the other hand, $L j P H A N b$ expression was continually confined to the adaxial domain of the incipient leaflet primordia in $\mathrm{p} 2$ and maintained at the adaxial domain in $\mathrm{p} 3$. Then it gradually dispersed in leaf primordia of $\mathrm{p} 4$ and $\mathrm{p} 5$ (Fig. $2 \mathrm{D}, \mathrm{E})$. However, expression of $\angle j P H A N b$ was also found at the base of leaflets from $\mathrm{P} 4$, and this strong expression still be seen at the leaflet base in much older leaflets (Fig. 2E). During the reproductive developmental stage, $\angle j P H A N a$ was expressed in the bracts and developing flower organs (Fig. 2C) whereas no LjPHANb transcript was detected in the floral organs (Fig. 2F). Taken together, expression pattern of $\angle j P H A N a$ is similar to PHAN in $A n$ -
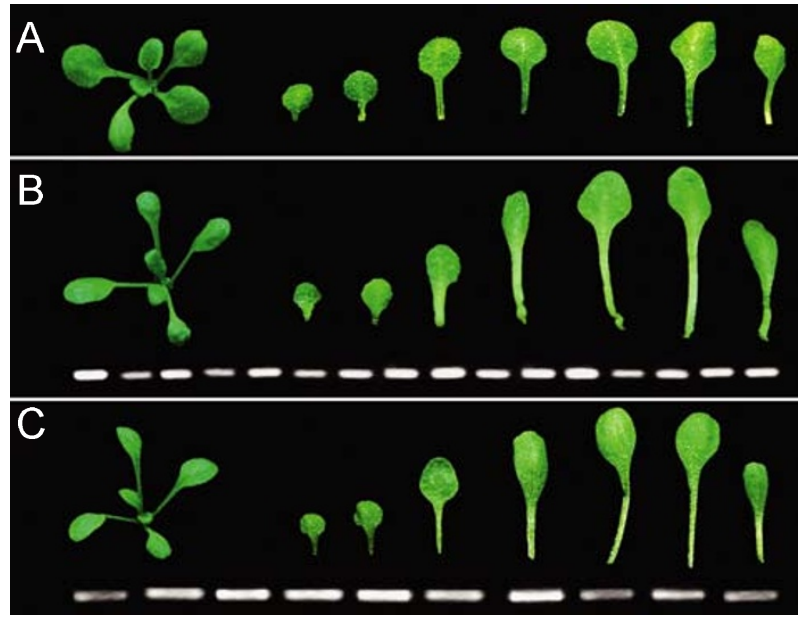

Fig. 3 Phenotypes of transgenic Arabidopsis lines overexpressing $\angle j P H A N a$ or LjPHANb. (A)Transgenic Arabidopsis transformed with empty vector; (B) $35 S:: L j P H A N a$ transgenic Arabidopsis and PCR confirmation of LjPHANa insertion; (C) $35 S: \because L j P H A N b$ transgenic Arabidopsis and PCR confirmation of $L j P H A N b$ insertion.

tirrhinum and AS1 in Arabidopsis [5, 13], whose expression is detected in both compound leaves and floral organs whereas $\angle j P H A N b$ is only expressed in compound leaf primordia and leaflet primordia, indicating that the $\angle j P H A N b$ gene plays a more specific role restricted to compound leaf development. 
A

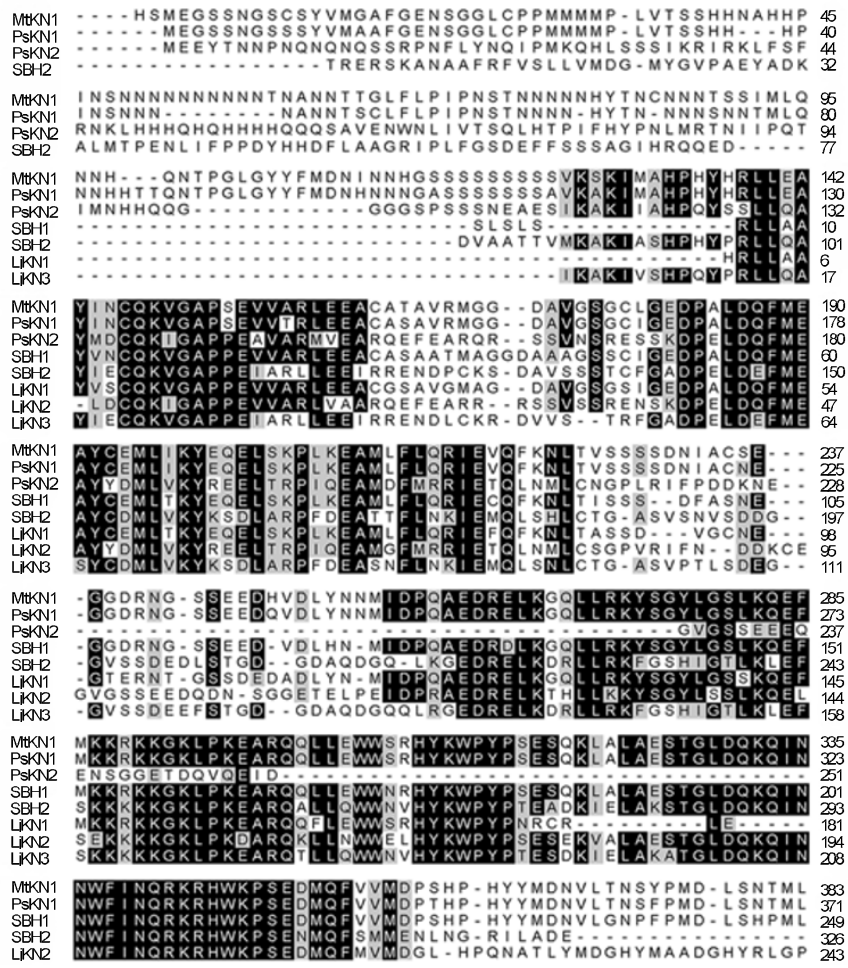

B

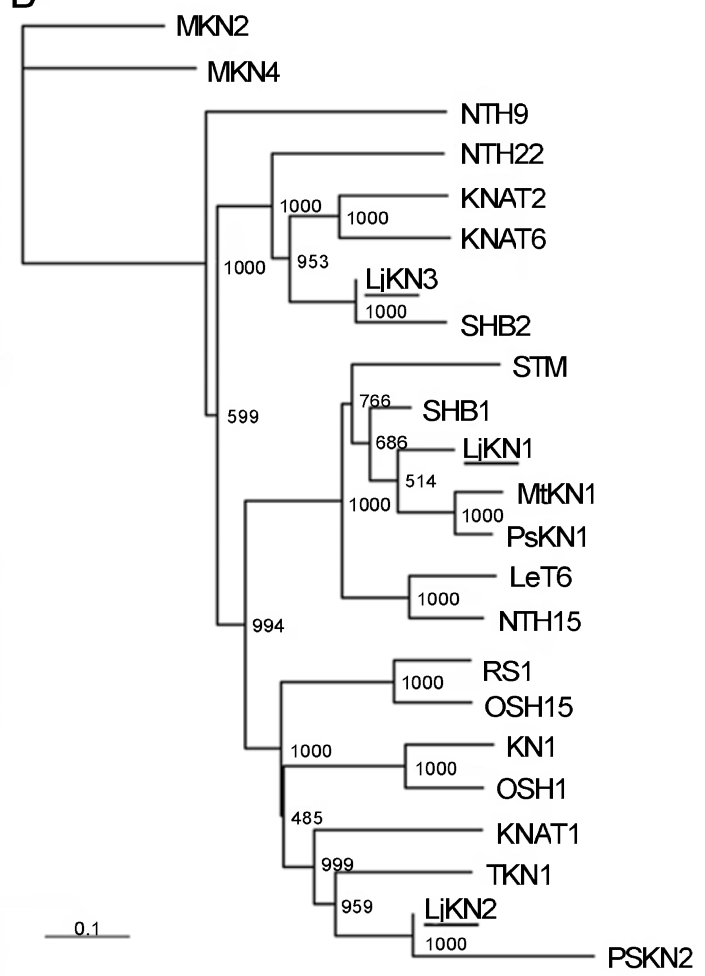

Fig. 4 Analysis of KNOXI genes in L. japonicus. (A) Multiple alignment of KNOXI proteins or fragments; residues identical in five of the proteins are shown in black. (B) A phylogenetic tree of KNOXI proteins based on Neighbour Joining method.

\section{Overexpression of $L j P H A N a$ and $L j P H A N b$ in Arabidopsis}

To investigate biochemical functions of the LjPHANa and LjPHANb proteins, we constructed plasmids containing $L j P H A N a$ or $L j P H A N b$ ORF driven by the $35 S$ promoter respectively, and transformed wild type Arabidopsis (Landsberg erecta ecotype). 16 independent transgenic lines for $L j P H A N a$ and 10 independent transgenic lines for $L j P H A N b$ were obtained. Similar leaf phenotypes in various extents were observed in T1 seedlings of transgenic plants transformed with either $35 S:: L j P H A N a$ or $35 S:$ : $L j P H A N b$, but not in T1 seedlings of transgenic plants transformed with empty vectors. The phenotypes observed in the transgenic lines were segregated well with the transgenes in the manner of either single or multi transgene insertions (data not shown). These transgenic plants typically displayed elongated leaves with narrower, longer blades and longer petioles (Fig. 3B, C), as compared with the leaves of wild type Landsberg erecta. The transgenic phenotypes mimicked to the ones of a previous study, in which the overexpression of $R S 2$ and $A S 1$ in Arabidopsis caused narrower leaves with longer petioles [37]. These results suggested that the function of either LjPHANa or
LjPHANb should be the same as other PHAN orthologs at protein level.

\section{Expression patterns of $K N O X I$ genes in $L$. japonicus}

Previous studies showed PHAN-KNOXI interaction in pea is similar to that in Arabidopsis [30]. To investigate whether it is the same case in L. japonicus, we isolated KNOXI genes and examined their expression patterns by RNA in situ hybridization.

Three KNOXI gene fragments were isolated from vegetative apex cDNA and named $L j K N 1, L j K N 2$ and $L j K N 3$, all belonging to the $K N O X I$ subgroup of $K N O X$ gene family. Sequence analysis and phylogenetic tree indicated that $L j K N 1$ shared high similarity with $P S K 1$ in pea and STM in Arabidopsis; LjKN2 resembled to PSKN2 in pea and KNAT1 in Arabidopsis; and LjKN3 was similar to KNAT2 or KNAT6 in Arabidopsis (Fig. 4A, B).

In wild-type plants, $L j K N 1$ and $L j K N 3$ were simultaneously expressed in the shoot apical meristems (SAM) and the axillary meristems (Fig. 5A, D) during the vegetative and reproductive stages, provascular tissue, inflorescences (Fig. 5A, D), floral meristems (Fig. 5B, E) and indeterminate tissues of developing floral buds (Fig. 5C, 


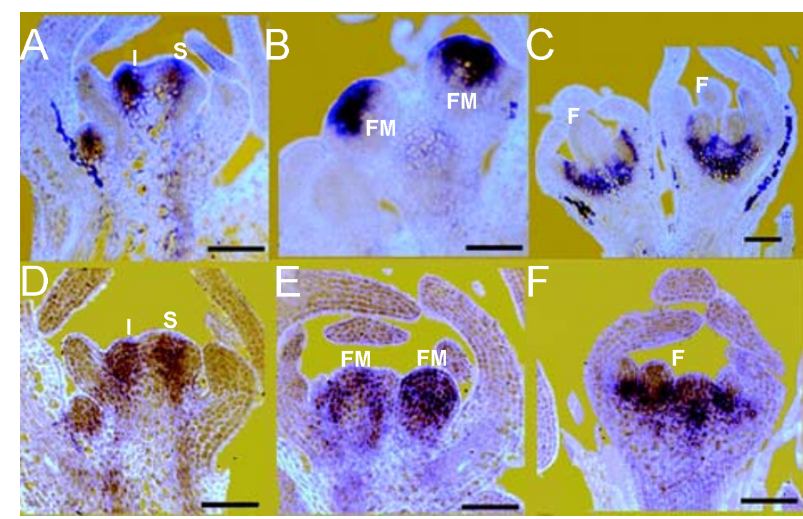

Fig. 5 Expression patterns of $L j K N$ genes in L. japonicus. (A-C) Expression patterns of $L j K N 1$ in SAM and inflorescence meristem (A), floral meristems (B), developing floral buds (C). (D-F) Expression patterns of $L j K N 3$ in SAM and inflorescence meristem (D), floral meristems (E), developing floral bud (F). S: SAM; I: inflorescence meristem; FM: floral meristem; F: floral bud. Scale bar $=50 \mu \mathrm{m}$.

F). However, some differences were found: the signal for $L j K N 1$ was always stronger and in much restricted area near the central zone of SAM, but the one for $L j K N 3$ was generally weaker and more dispersed. No $L j K N 1$ and $L j K N 3$ transcripts were detected in the compound leaf primordia or leaflet primordia at any stage, consistent with previous reports about no expression of KNOXI genes in the compound leaf primordia in pea $[24,30]$. On the other hand, we failed to detect the expression of $L j K N 2$ in any tissues at both vegetative and reproductive stages by RNA in situ hybridization.

Genbank Accession Numbers: MKN2 (Physcomitrella patens), AF285147; MKN4 (Physcomitrella patens), AF284817; STM (Arabidopsis), ATU32344; KNAT1 (Arabidopsis), AF482994; KNAT2 (Arabidopsis), ATU14175; KNAT6 (Arabidopsis), NM160620; PsKN1 (pea), AF080104; MtKN1 (Medicago trucatula), AF308454; PsKN2 (pea), AF080105; SBH1 (soybean), L13663; KN1 (maize), AY312169; RS1 (maize), L44133; LeT6 (tomato), AF000141; TKN1 (tomato), U33247; OSH1 (rice), D16507; OSH15 (rice), AB016071; NHT15 (tobacco), AB004785.1; NTH22 (tobacco), AB025715; NTH9 (tobacco), AB025713; LjKN1 (L. japonicus), AY790246; LjKN2 (L. japonicus), AY790247; LjKN3 (L. japonicus), AY790248.

\section{The reduced leaflets mutants with decreased adaxial identity of compound leaves}

We tried to identify mutants with decreased adaxial identity of compound leaves in L. japonicus from ethyl methanesulfonate (EMS) mutant collection. Two mutants with abnormal compound leaves were identified, one being isolated from the SL256 mutated line [39] and the other from the D0013 mutated line (unpublished data). In both cases, the F1 backcross progeny had the wild-type phenotype, and the F2 progeny yielded phenotypes at a Mendelian segregation ratio of 3:1 (132 wild type: $46 \mathrm{mu}-$ tant for $S L 256, P<0.5$; 117 wild type: 42 mutant for $D 0013$, $P<0.5$ ), indicating that the phenotype of each mutant was caused by a single recessive mutation. The two mutants exhibited nearly identical phenotypes and the most distinct defects were abnormal compound leaves with narrow leaflets and reduced leaflet numbers. According to their phenotypes, we named the two mutants reduced leaflets 1 (rell) and reduced leaflets 3 (rel3). Genetic analysis showed that the corresponding loci Rell and Rel3 were not allelic and none of them were linked to the $L j P H A N \mathrm{~s}$ (unpublished data).

In wild-type L. japonicus, nodes from one to five produce compound leaves with three or four leaflets. Usually from the sixth node, plants produce mature compound leaves containing five visible leaflets: one top leaflet, two lateral leaflets and two basal leaflets (Fig. 6A). In contrast, most of the compound leaves in both rell and rel3 were composed of three narrow leaflets, regardless of the node (Fig. 6B, C). In both mutants, the leaflets were narrower and thicker than wild type; the proximal parts of the leaflets being rod-like, and the leaflet edges slightly curled up. The most severely affected leaflets were not completely expanded, displaying the needle shape (Fig. 6B, C).

We further compare the compound leaf development in both wild type and mutant plants by scanning electron microscopy (SEM). In the wild type plants, the leaflet primordia basipetally initiated from the compound leaf primordia, leading to formation of the 5 leaflet primordia (Fig. 6D). In rell and rel3 mutants, fewer leaflet primordia were initiated. Once the top leaflet primordia initiated, the compound leaf primordia normally stopped initiating new leaflet primordia in the mutants (Fig. 6E, F).

SEM analysis was also conducted to analyze the epidermis of compound leaves in rel mutants. The adaxial surface of a fully expanded wild-type leaflet consisted of the multiangular cells (Fig. 6G), whereas the abaxial surface possessed multiangular cells and elongated narrow cells located in the middle part of the leaflets (Fig. 6J). The boundary between the adaxial and abaxial surfaces also consisted of several rows of elongated cells. By comparison, the adaxial and abaxial epidermis of leaflets in rell and rel3 were similar to each other, but drastically different from wild type. The abaxial surface of the mutant leaflets, especially on the proximal parts, was only composed of elongated narrow cells (Fig. 6K, L) that were 

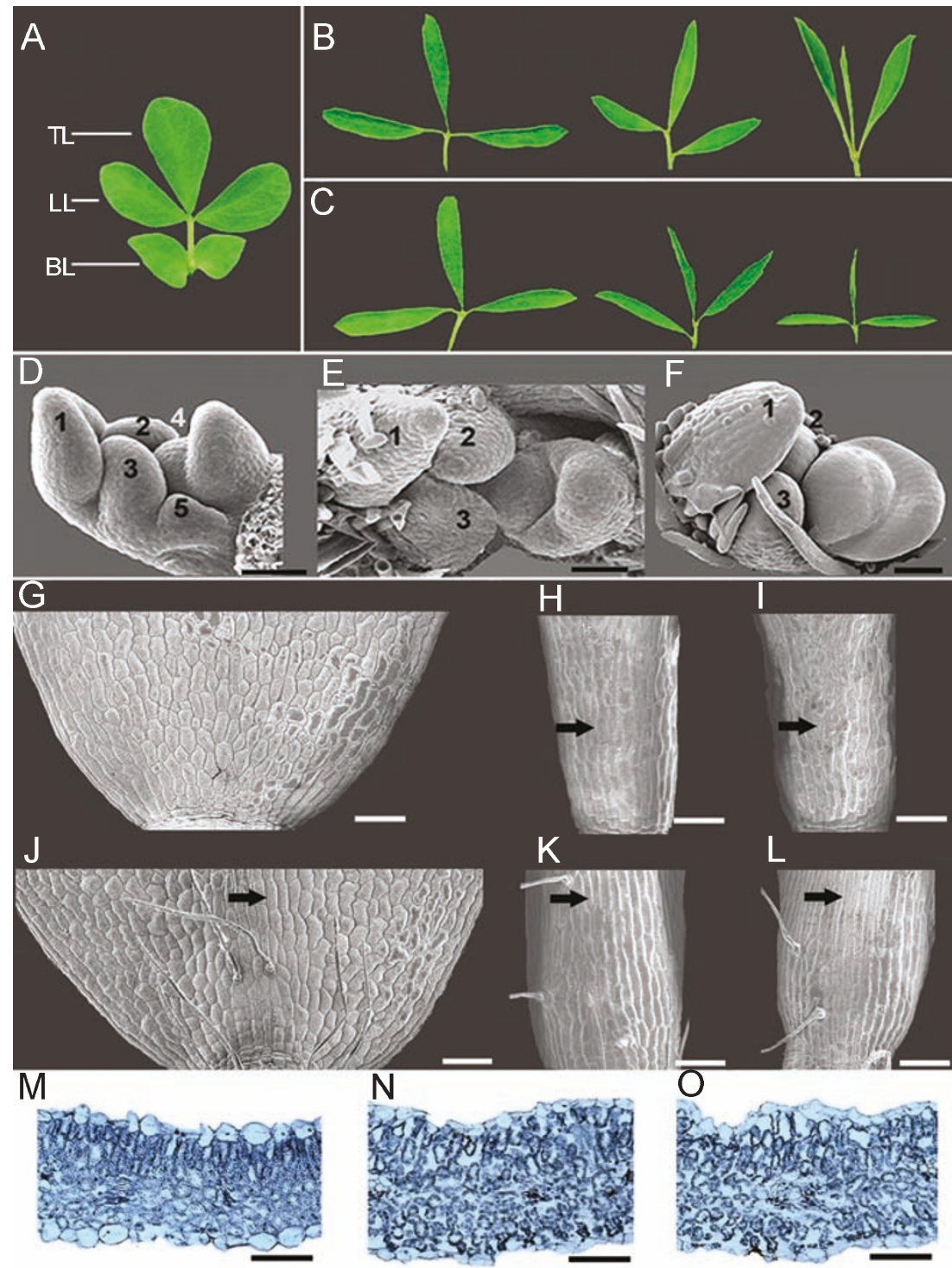

$\mathrm{H}$

Fig. 6 Analysis of abnormal compound leaves in the rel mutants. (A-C) Comparison of compound leaves among wild type (L. japonicus, Gifu ecotype) (A), rell (B), and rel3 (C); TL: top leaflet; LL: lateral leaflet; BL: bottom leaflet. (D-F) Comparison of leaflet initiation by SEM among wild type (D), rell (E) and rel3 (F); Numbers indicate the sequential initiation of leaflet primordia (The fourth leaflet primordia of wild type is shielded by the next compound leaf primordia). (G-I) Comparison of adaxial epidermis by SEM among wild type (G), rell (H) and rel3 (I). (J-L) Comparison of abaxial epidermis by SEM among wild type (J), rell (K) and rel3 (L); only the proximal parts being showed, in which arrow indicates the elongated cells. (M-O) Histological transverse sections of leaflet blade among wild type (L. japonicus, Gifu ecotype) $\mathbf{( M )}$, rell (N), and rel3 (O). Scale bar $=50 \mu \mathrm{m}$.

also proliferating to the adaxial surface (Fig. 6H, I); resulting in narrowed and approximately round leaflet bases. The appearance of elongated narrow cells on the proximal part of the adaxial surface (Fig. $6 \mathrm{H}, \mathrm{I}$ ) in mutant leaflets suggests that adaxial identity was reduced in $\mathrm{rell}$ and $\mathrm{rel} 3$ compound leaves.

Sections of leaflets further revealed reduced adaxialabaxial polarity in mutant compound leaves. On wild type leaf sections, elongated palisade mesophyll cells are lo- cated in the adaxial region and round spongy mesophyll cells in the abaxial region (Fig. 6M). Whereas in both mutants, palisade mesophyll in the adaxial domain became almost round and difficult to distinguish from spongy mesophyll in the abaxial domain, as well as mesophyll cells were arranged sparsely and disorderly as compared with wild type (Fig. 6N, O). These results confirmed that the adaxial identity was decreased in the compound leaves in rell and rel3 mutants. 


\section{Altered expression patterns of $\mathrm{LjPHAN}$ genes in rel mutants}

The Rel1 and Rel3 loci were not linked to LjPHANa and $L j P H A N b$. However, the most prominent phenotype of the rell and rel3 mutants was decreased adaxial-abaxial polarity and reduced complexity of compound leaves, suggesting the possible involvement of abnormal PHAN-like gene function. Thus, we further characterized the relationship between the rel mutant phenotypes and the PHANlike genes by examining the expression patterns of $L j P H A N a$ and $L j P H A N b$ in the mutants using RNA in situ hybridization and reverse transcription-polymerase chain reaction ( $R T-P C R)$.

The results of RT-PCR revealed that the expression levels of $L j P H A N a$ and $L j P H A N b$ were slightly increased in both vegetative shoot tips and matured leaves of rell and rel3 mutants as compared with wild type, suggesting that the overall expression level of both $L j P H A N$ s was not dramatically changed in the mutants (data not shown).

However, RNA in situ hybridization showed that expression patterns of $L j P H A N a$ and $L j P H A N b$ were altered in rell and rel3 mutants in comparison with wild type. In both mutants, $L j P H A N a$ was diffusely expressed throughout $\mathrm{p} 1$ compound leaf primordia (Fig. 7B, C). In p2, the $L j P H A N a$ signal continued to be dispersed in leaflet primordia without the normally observable stronger expression at the middle region of leaflets (Fig. 7B, C). Similarly, $L j P H A N b$ expression was also diffused throughout P1 compound leaf primordia (Fig. 7E, F). After leaflet initiation, $L j P H A N b$ expression was still dispersed in the newly initiated leaflet primordia of p2 and p3 (Fig. 7E, F). After leaflets expanded, the expression domains for both $\angle j P H A N a$ and $L j P H A N b$ were progressively confined to the adaxial side at the distal part of the leaflets (Fig. 7B, C, E). The expression of $L j P H A N b$ at the bases of the leaflet primordia was much weaker or lost in both rel mutants (Fig. 7H, I).

In contrast, expression patterns of $L j K N 1$ and $L j K N 3$ displayed no significant changes in the rell and rel3 mutants. Like for wild type, neither of these genes was detectable by in situ hybridization, in any compound leaf primordia and leaflet primordia of the rell and rel3 mutants (Fig. 7K, L, N, O). Also, transcripts of $L j K N 1$ and $L j K N 3$ could be detected by RT-PCR from the vegetative shoot tips in wild type and in the rel mutants as well, without significant difference between them (data not shown).

\section{DISCUSSION}

\section{$P H A N$-like gene duplication in $L$. japonicus}

Gene duplication has occurred widely in the genomes of disparate life forms, contributing greatly to evolution and diversity and elaboration of basic developmental form.
Except for genome duplication, gene duplication often occurs in three ways: unequal crossing over, retroposition and chromosomal rearrangement [40].

We isolated two PHAN-like genes in L. japonicus genome, $L j P H A N a$ and $L j P H A N b$, and found them in a tandem arrangement on chromosome 3 , which strongly indicated that the two genes likely originated from gene duplication caused by unequal crossing over. As duplication of PHAN gene had not been reported in species other than L. japonicus, we investigated whether it might have also occurred in other legumes. Database searching allowed us to identify a similar pair of $P H A N$-like genes in soybean and genomic Southern blotting showed that there was possibility of more than one copy of PHAN-like gene in M. trucatula and Pisum sativum genomes. Phylogenetic analysis of PHAN proteins revealed that duplication of $P H A N$-like genes possibly was an ancient duplication and most likely occurred before the species divergence of legumes. Consistently, it has been suggested unaffected tendrils and stipules never being radialized in pea $c r i$ mutants, are likely to result from redundancy of $P H A N$ like genes [30]. Genome sequencing data in M. trucatula and other papilionoid legumes will be required to adequately address this possibility.

Sequence analysis indicated that $L j P H A N a$ shared higher similarity with $P H A N$ ortholog in pea than with $L j P H A N b$. In the phylogenetic tree based on putative sequences of PHAN-like proteins, LjPHANa and LjPHANb rooted in different clades. Expression patterns of both LjPHANs also differ from each other. However, the transgenic phenotypes caused by overexpression of either LjPHANa or LjPHANb in Arabidopsis, resembled to each other and to the phenotypes of $35 S:: A S 1$ [37], which suggested that both LjPHAN proteins could exert similarly conserved biochemical functions as AS1 in Arabidopsis. These results suggested that the duplicated $P H A N$-like genes were functional and the biological function difference between them, if there is any, was likely to be the outcome of their specific expression patterns.

\section{Divergent functions of $L j P H A N$ genes in $L$. japonicus compound leaf development}

As the previous research showed, like for simple leaf formation, correct establishment of adaxial-abaxial polarity is important for compound leaf development. PHANlike genes were found to reserve functions in setting up adaxial identity in compound leaf primordia and leaflet primorida $[19,27]$. It has been shown that adaxial-abaxial polarity of the compound leaves was reduced in pea mutant cri and CRI was a pea ortholog of PHANTASTICA [30].

During L. japonicus compound leaf development, 

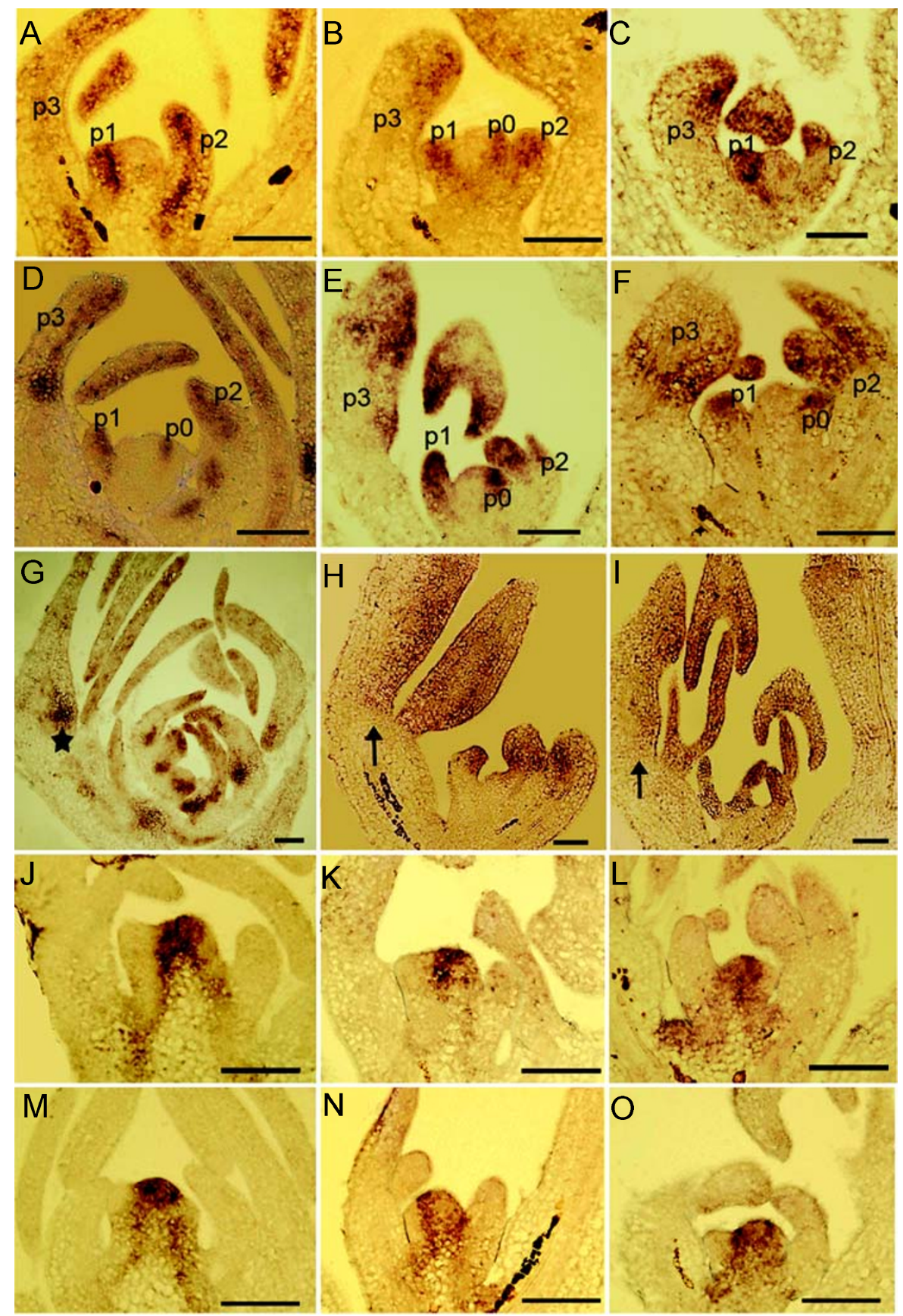

Fig. 7 Expression patterns of $L j P H A N \mathrm{~s}$ and $L j K N \mathrm{~s}$ in wild type and rel mutants. (A-C) Expression patterns of $L j P H A N a$ in wild type (A), rell (B) and rel3 (C). (D-F) Expression patterns of $L j P H A N b$ in wild type (D), rell (E) and rel3 (F). (G-I) Expression patterns

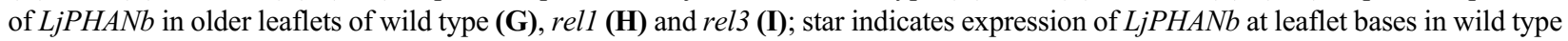
(G), and arrows indicate no expression of $L j P H A N b$ at leaflet bases in rel mutants (H, I). (J-L) Expression patterns of $L j K N 1$ in wild type (J), rell (K) and rel3 (L). (M-O) Expression patterns of LjKN3 in wild type (M), rell $\mathbf{( N )}$ and rel3 (O). Scale bar $=50 \mu \mathrm{m}$.

$\angle j P H A N a$ and $L j P H A N b$ displayed different expression patterns. Like all $P H A N$-like genes described so far, both LjPHAN transcripts were firstly detected in leaf founder cells ( $\mathrm{p} 0)$, which was believed to be responsible for leaf determination $[5,13,16,18]$. In $p 1$ compound leaf primordia, they were expressed with stronger level at the adaxial domain, suggesting their functions in establishment of adaxial identity in compound leaf primordia. After leaf- 
let primordia initiated, $L j P H A N a$ was detected with stronger level at the middle region of leaflet primordia whereas $\angle j P H A N b$ expression continued at the adaxial domain of leaflet primordia where it perhaps helped specify adaxial identity of leaflets. In expanded leaflets, $L j P H A N b$ displayed a unique expression pattern at bases of leaflets, possibly involved in stabilizing the boundary between leaflets and petiolules. Consistently, expression of $\mathrm{LjPHANa}$ was detected in both compound leaf and flower, but the one of $L j P H A N b$ was exclusive from flower, strongly indicating that $\angle j P H A N b$ may not play an important in the floral development.

Therefore, based on their expression pattern difference, $\angle j P H A N b$ and $L j P H A N a$ should be defined as paralogs with divergent functions and play different roles in $L$. japonicus compound leaf development. Recent studies showed that evolutionary switch of some basic morphological forms could only involve the regulatory regions of some master genes. In the case of evolutionary switch from teosinte to maize, the regulatory region of $T b 1$, a gene encoding for a putative transcription factor, was changed but not in the coding region of the gene [41, 42]. It is possible that the similar mechanism occurred in $L$. japonicus, the divergent biological functions of duplicated LjPHAN genes depending on their distinct expression patterns rather than their protein differences.

The morphology of compound leaf in L. japonicus is simpler than the one of pea, since it consists of five leaflets with the same identity (Fig. 6A). However, functions of PHAN-like genes are well conserved; both LjPHANS being expressed in compound leaf primordia indicated that they likely reserve the functions in establishment of adaxial identity. On the other hand, the expression pattern difference between $\angle j P H A N a$ and $\angle j P H A N b$ suggested that in L. japonicus, adaxial identity of compound leaves could be specified at two levels, adaxial identity of compound leaf primordia built by both $L j P H A N$ genes while adaxial identity of leaflet primordia only by LjPHANb.

\section{The functions of Rel1 and Rel3 in compound leaf development of $L$. japonicus}

It is believed that compound leaf specification consists of two aspects, leaflet initiation and leaflet pattern formation. Correct leaflet initiation determines the leaflet number and placement, which is regulated by marginal meristem maintenance and the adaxial-abaxial polarity of the compound leaf primordia [1, 19, 25]. Although KNOXI and PHANlike genes have been shown to be involved in the process $[19,25]$, there are likely other factors regulating compound leaf development. In this study, we present novel candidates Rel1 and Rel3, which induce disturbances in compound leaf development when mutated.
The phenotypes of the rell and rel3 mutants are highly similar to each other, suggesting that Rell and Rel3 genes might function in the same genetic pathway. The rell and rel3 mutants displayed abaxialization of adaxial epidermis and palisade mesophyll of leaflets (Fig. 6G-O), indicating decreased adaxial identity in compound leaves. Although Rell and Rel3 loci were not linked to the LjPHANs, the abnormalities of compound leaves in both mutants were associated with altered expression patterns of $\angle j P H A N a$ and $L j P H A N b$, the newly isolated $P H A N$-like genes in $L$. japonicus. In rel mutants, both $L j P H A N$ transcripts were dispersed throughout compound leaf primordia and leaflet primordia, which lost their distinct expression patterns (Fig. 7B, C, E, F, H, I). As a result, adaxial epidermis and palisade mesophyll of compound leaves were differentiated improperly. Only in the expanded leaflet primordia, both $L j P H A N$ expressions had gradually been confined to the adaxial domain in the distal parts, which was also consistent with mutant phenotypes that the most leaflets still have narrow blades. The correlation between expression pattern and compound morphology in both wild type and mutants further strongly support the notion that $L j P H A N a$ and $\angle j P H A N b$ genes played important roles in adaxial identity specification in the development of L. japonicus compound leaves.

These data also suggest that Rel loci are epistatic to LjPHANs in regulating their spatial expression pattern and controlling the adaxial-abaxial polarity during compound leaf development in L. japonicus, although the clear relationship between them needs to be further investigated. Apart from the defects in compound leaf development, the rel mutants also exhibited abnormalities in cotyledons, floral development and stem vascular pattern formation (data not shown). The pleiotropic developmental defects in both mutants suggest that Rel loci are possibly involved in a fundamental regulation and responsible for controlling many aspects of $L$. japonicus ontogeny.

\section{The relationship between $L j K N$ genes and $L j P H A N$ genes in $L$. japonicus}

Previous studies in pea have questioned the role of $K N O X I$ genes in compound leaf development in papilionoid legumes [24]. However, recent study showed that the PHAN$K N O X I$ relation in pea is like that in Arabidopsis rather than in tomato, PSPHAN suppressing PsKN2 expression in wild-type compound leaf primordia [30].

In L. japonicus, expression of $L j K N 1$ and $L j K N 3$ was restricted to the SAM and exempt from the compound leaf primordia and leaflet primordia. The mutually exclusive expression patterns between $L j K N s$ and $L j P H A N s$ in wild type might suggest that the duplicated $P H A N$-like genes could be involved in repressing the expression of KNOXI 
genes in compound leaf primordia, being consistent with $P H A N-K N O X I$ relation in pea [30]. Considering conservation of re-establishment of KNOXI expression in compound leaf primordia among species from fern to angiosperm [25], we could speculate KNOXI genes were expressed in the ancient compound leaf primordia, which have been maintained through evolution in most species but repressed by redundant expression of $P H A N$-like genes after gene duplication in L. japonicus and possibly in other papilionoid legumes. Therefore, it is likely that the duplication of PHAN-like genes contributes to the special developmental mechanism in legumes compound leaves. Loss of function mutants of $\angle j P H A N a$ and $L j P H A N b$ remain to be identified to explore these possibilities.

On the other hand, other factors, such as Rell and Rel3, are expected to play important roles in the mechanism. Based on the results of in situ hybridization and RT-PCR, we found that $L j P H A N$ genes were mainly affected on spatial expression domain whereas expression of $L j K N$ genes was not altered, when Rell or Rel3 was mutated. This might reflect the specific function of Rel genes as the regulators for LjPHANs. With cloning of both Rell and Rel3 in the near future, it will provide a clear insight into the interaction among Rels, $L j P H A N \mathrm{~s}$ and $L j K N \mathrm{~s}$, and lead to a better understanding for the molecular mechanism controlling compound leaf development in legumes.

\section{ACKNOWLEDGEMENTS}

The authors thank Dr. Hai HUANG for the vectors used in Arabidopsis transformation, and Dr. Bernard J. CARROLL for helpful revision. This research was financially supported by the National Natural Science Foundation of China (Grant No. 30430330).

Received, May 31, 2005

Revised, July 28, 2005

Accepted, Aug 11, 2005

\section{REFERENCES}

1 Bharathan G, Sinha NR. The regulation of compound leaf development. Plant Physiol 2001; 127:1533-8.

2 Micol J, Hake S. The development of plant leaves. Plant Physiol 2003; 131:389-94.

3 Bowman JL, Eshed Y, Baum SF. Establishment of polarity in angiosperm lateral organs. Trends Genet 2002; 18:134-41.

4 Bohmert K, Camus I, Bellini C, et al. AGOI defines a novel locus of Arabidopsis controlling leaf development. EMBO J 1998; 17: 170-80.

5 Waites R, Selvadurai HRN, Oliver IR, Hudson A. The Phantastica gene encodes a MYB transcription factor involved in growth and dorsoventrality of lateral organs in Antirrhinum. Cell 1998; 93: 779-89.

6 Lynn K, Fernandez A, Aida M, et al. The PINHEAD/ZWILLE gene acts pleiotropically in Arabidopsis development and has overlapping functions with the ARGONAUTE1 gene. Development 1999; 126:469-81.

7 McConnell JR, Emery J, Eshed Y, et al. Role of PHABULOSA and $P H A V O L U T A$ in determining radial patterning in shoots. Nature 2001; 411:709-13.

8 Siegfried KR, Eshed Y, Baum SF, et al. Members of the YABBY gene family specify abaxial cell fate in Arabidopsis. Development 1999; 126:4117-28.

9 Kerstetter RA, Bollman K, Taylor RA, et al. KANADI regulates organ polarity in Arabidopsis. Nature 2001; 411:706-8.

10 Waites R, Hudson A. Phantastica: a gene required for dorsoventrality of leaves in Antirrhnum majus. Development 1995; 121: 2143-54.

11 Tsiantis M, Schneeberger R, Golz JF, et al. The maize rough sheath2 gene and leaf development programs in monocot and dicot plants. Science 1999; 284:154-6.

12 Timmermans MCP, Hudson A, Becraft PW, Nelson T. Rough sheath2: A $M y b$ protein that represses knox homeobox genes in maize lateral organ primordia. Science 1999; 284:151-3.

13 Byrne ME, Barley R, Curtis M, et al. Asymmetric leaves 1 mediates leaf patterning and stem cell function in Arabidopsis. Nature 2000; 408:967-71.

$14 \mathrm{Xu} \mathrm{L}, \mathrm{Xu}$ Y, Dong A, et al. Novel as 1 and as 2 defects in leaf adaxial-abaxial polarity reveal the requirement for $A S Y M M E T$ RIC LEAVESI and 2 and ERECTA functions in specifying leaf adaxial identity. Development 2003; 130:4097-107.

15 Schneeberger R, Tsiantis M, Freeling M, Langdale JA. The rough sheath 2 gene negatively regulates homeobox gene expression during maize leaf development. Development 1998; 125:2857-65.

16 McHale NA, Koning RE. PHANTASTICA regulates development of the adaxial mesophyll in Nicotiana leaves. Plant Cell 2004; 16: 1251-62.

17 Ori N, Eshed Y, Chuck G, et al. Mechanisms that control knox gene expression in the Arabidopsis shoot. Development 2000; 127:5523-32.

18 Harrison CJ, Corley SB, Moylan EC. Independent recruitment of a conserved developmental mechanism during leaf evolution. Nature 2005; 434:509-14.

19 Kim M, McCormick S, Timmermans M, Sinha N. The expression domain of PHANTASTICA determines leaflet placement in compound leaves. Nature 2003; 424:438-43.

20 Lincoln C, Long J, Yamaguchi J, et al. A knotted1-like homeobox gene in Arabidopsis is expressed in the vegetative meristem and dramatically alters leaf morphology when overexpressed in transgenic plants. Plant Cell 1994; 6:1859-76.

21 Hareven D, Gutfinger T, Parnis A. The making of a compound leaf: genetic manipulation of leaf architecture in tomato. Cell 1996; 84:735-44.

22 Parnis A, Cohen O, Gutfinger T, et al. The dominant developmental mutants of tomato, Mouse-ear and Curl, are associated with distinct modes of abnormal transcriptional regulation of a Knotted gene. Plant Cell 1997; 9:2143-58.

23 Janssen BJ, Lund L, Sinha N. Overexpression of a homeobox gene, LeT6, reveals indeterminate features in the tomato compound leaf. Plant Physiol 1998; 117:771-86.

24 Hofer J, Gourlay C, Michael A, Ellis TH. Expression of a class 1 knottedl-like homeobox gene is down-regulated in pea compound leaf primordia. Plant Mol Biol 2001; 45:387-98.

25 Bharathan G, Goliber TE, Moore C, et al. Homologies in leaf 
form inferred from $K N O X I$ gene expression during development. Science 2002; 296:1858-60.

26 Koltai H, Bird DM. Epistatic repression of PHANTASTICA and class I KNOTTED genes is uncoupled in tomato. Plant $\mathrm{J} 2000$; 22:455-9.

27 Kim M, Pham T, Hamidi A, et al. Reduced leaf complexity in tomato wiry mutants suggests a role for PHAN and KNOX gene in generating compound leaves. Development 2003; 130:440515.

28 Hofer J, Turner L, Hellens R, et al. UNIFOLIATA regulates leaf and flower morphogenesis in pea. Curr Biol 1997; 7:581-7.

29 Taylor S, Hofer J, Murfet I. Stamina pistilloida, the Pea Ortholog of Fim and UFO, is required for normal development of flowers, inflorescences, and leaves. Plant Cell 2001; 13:31-46.

30 Tattersall AD, Turner L, Knox MR, et al. The Mutant crispa Reveals Multiple Roles for PHANTASTICA in Pea Compound Leaf Development. Plant cell 2005; 17:1046-60.

31 Hall TA. BioEdit: a user-friendly biological sequence alignment editor and analysis program for Windows 95/98/NT. Nucl Acids Symp Ser 1999; 41:95-8.

32 Thompson JD, Gibson TJ, Plewniak F, et al. The CLUSTALX windows interface: flexible strategies for multiple sequence alignment aided by quality analysis tools. Nucleic Acids Res 1997; 25: 4876-82

33 Page RDM. TREEVIEW: An application to display phylogenetic trees on personal computers. Comput Appl Biosci 1996; 12: $357-8$.

34 Alvarez J, Guli CL, Lu XH, Smyth DR. Terminal flower: a gene affecting inflorescence development in Arabidopsis thaliana. Plant J 1992; 2:103-16.

35 Jackson D, Veit B, Hake S. Expression of maize KNOTTED1 related homeobox genes in the shoot apical meristem predicts patterns of morphogenesis in the vegetative shoot. Development 1994; 120:405-13.

36 Bradley D, Carpenter R, Sommer H, et al. Complementary floral homeotic phenotypes result from opposite orientations of a transposon at the plena locus of Antirrhinum. Cell 1993; 72:8595.

37 Theodoris G, Inada N, Freeling M. Conservation and molecular dissection of ROUGH SHEATH2 and ASYMMETRIC LEAVES1 function in leaf development. Proc Natl Acad Sci U S A 2003; 100:6837-42.

38 Nakamura Y, Kaneko T, Asamizu E, et al. Structural analysis of a Lotus japonicus genome. II. Sequence features and mapping of sixty-five TAC clones which cover the $6.5 \mathrm{Mb}$ regions of the genome. DNA Res 2002; 9:63-70.

39 Perry JA, Wang TL, Welham TJ, et al. A tilling reverse genetics tool and a web-accessible collection of mutants of the legume Lotus japonicus. Plant Physiol 2003; 131:866-71.

40 Zhang J. Evolution by gene duplication: an update. Trends Ecol Evol 2003; 18:292-8.

41 Doebley J, Stec A, Hubbard L. The evolution of apical dominance in maize. Nature 1997, 386:485-8.

42 Wang RL, Stec A, Hey J, Lukens L, Doebley J. The limits of selection during maize domestication. Nature, 1999, 398:2369. 